\title{
A Comparative Study of Higher Order Bragg Gratings: Coupled-Mode Theory Versus Mode Expansion Modeling
}

\author{
H. Wenzel, R. Güther, A. M. Shams-Zadeh-Amiri, Member, IEEE, and P. Bienstman
}

\begin{abstract}
The modal reflectivity and loss of lamellar diffraction gratings to be used in distributed-feedback and distributed-Bragg reflector lasers were computed in dependence of wavelength, duty cycle and Bragg order. Different methods based on the approximate coupled-mode theory and and the exact bidirectional mode expansion modeling were compared and a good mutual agreement was found. The slab Green's function needed to compute the coupling coefficients can be approximated by that of a homogeneous unbounded medium with sufficient accuracy.
\end{abstract}

Index Terms-Bragg grating, mode matching, radiation losses.

\section{INTRODUCTION}

$\mathbf{L}$ AMELLAR diffraction gratings integrated into slab waveguides (so-called Bragg waveguide gratings) found widespread applications in distributed-feedback (DFB) and distributed Bragg-reflector (DBR) lasers, either as edge or as surface emitters. The propagation of electromagnetic waves in these structures is essentially of two-dimensional nature. Furthermore, the finite length of the gratings disturbs the one-dimensional periodicity. During the last years, higher order Bragg waveguide gratings have attracted again increasing interest because of the demand for high-power edge-emitting DFB and DBR lasers emitting at wavelengths below $1 \mu \mathrm{m}$. Due to their larger periods, they can be easier fabricated.

For the numerical simulation of these structures, a large variety of different models exists. Many models are based on the coupled-mode theory (CMT), which was firstly applied to DFB lasers in [1] and which was later improved in [2]. Within the CMT the mode field is expressed as an infinite summation of partial waves dictated by the Bloch-Floquet theorem. The results are coupled-mode equations for the amplitudes of the oppositely propagating waves directly interacting with the Bragg grating and an infinite set of equations for partial waves describing the radiation and evanescent fields. As outlined in [2], these fields lead to a modification of the coefficients entering the coupled-wave equations of [1].

Manuscript received April 3, 2005; revised August 30, 2005. This work was supported in part by Zukunftsfond Berlin and in part by the Flemish Fund for Scientific research (FWO-Vlaanderen).

H. Wenzel and R. Güther are with the Ferdinand-Braun-Institut für Höchstfrequenztechnik, Berlin 12489, Germany (e-mail: wenzel@fbh-berlin.de).

A. M. Shams-Zadeh-Amiri is with Photonami Corporation Richmond Hill, ON L4B 1E4, Canada.

P. Bienstman is with the Department of Information Technology, Ghent University, Ghent 9000 , Belgium.

Digital Object Identifier 10.1109/JQE.2005.859910

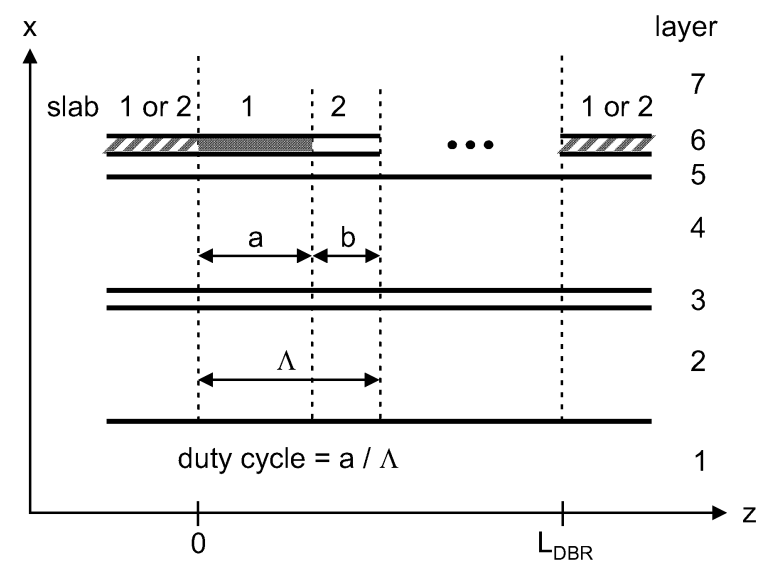

Fig. 1. Benchmark example.

Other models do not rely on the Bloch-Floquet theorem, but are based on a direct solution of Maxwell's equations. Some of these models based on the method of lines (MoL), bidirectional mode expansion or finite-difference time-domain (FDTD) methods were compared in [3] by calculating the modal reflectivity and transmittance of first-order Bragg waveguide gratings. A good mutual agreement was found.

It is well known [4] that due to the approximations involved in the derivation of the CMT it can not be applied to deeply etched gratings as considered in [3]. However, the question arises whether the CMT works for shallow gratings as typically used in index-coupled DFB and DBR lasers and especially whether the radiation loss of higher order gratings is correctly computed because a comparison of the more rigorous models with those based on the CMT is still missing.

In this paper, the modal reflectivity and transmittivity of first and higher order Bragg waveguide gratings are calculated using models based on the CMT [5] and the bidirectional mode expansion model CAMFR [6]. Thereby, an approximate solution for the partial waves first presented in [7] exploiting the free-space Green's function is corrected and generalized.

The paper is organized as follows. In Section II, the benchmark example is introduced. The numerical methods are described in Section III and the results are presented in Section IV.

\section{BENCHMARK EXAMPLE}

The Bragg waveguide grating under study schematically drawn in Fig. 1 consists of a periodic stack of two slab waveguides, named slab 1 and slab 2. Details of the thicknesses and 
TABLE I

THICKNESS $d$ AND REFRACTIVE INDICES $n$ OF THE CONSTITUENT SLAB WaVEGUIDES

\begin{tabular}{|c|c|c|c|}
\hline layer & $d / \mu \mathrm{m}$ & $n_{1}$ & $n_{2}$ \\
\hline 7 & 2.0 & \multicolumn{2}{|c|}{3.201} \\
\hline 6 & 0.04 & 3.523 & 3.201 \\
\hline 5 & 0.05 & \multicolumn{2}{|c|}{3.201} \\
\hline 4 & 0.25 & \multicolumn{2}{|c|}{3.218} \\
\hline 3 & 0.038 & \multicolumn{2}{|c|}{3.467} \\
\hline 2 & 0.25 & \multicolumn{2}{|c|}{3.218} \\
\hline 1 & 2.0 & \multicolumn{2}{|c|}{3.201} \\
\hline
\end{tabular}

refractive indexes of the two slabs are given in Table I. The only difference between the two slabs is caused by the variation of the refractive index of layer 6 .

For given Bragg order $N$ and reference (Bragg) wavelength $\lambda_{0}=980 \mathrm{~nm}$, the total length of the period of the grating is given by

$$
\Lambda=\frac{N \lambda_{0}}{2 n_{\mathrm{eff}}} .
$$

For given duty cycle $D$, the effective index $n_{\text {eff }}$ of the reference waveguide is obtained by averaging the dielectric function $\varepsilon(x, z) \equiv n^{2}(x, z)$ along $z$

$$
n_{\text {ref }}^{2}(x)=D n_{1}^{2}(x)+(1-D) n_{2}^{2}(x) .
$$

It varies between the effective indexes of the constituent slab waveguides $n_{\mathrm{eff} 2}=3.217063$ for $D=0$ and $n_{\text {eff } 1}=3.229026$ for $D=1$. Hence, the grating period is correspondingly changed between $\Lambda_{\max }=$ $N \times 152.312851 \mathrm{~nm}$ and $\Lambda_{\min }=N \times 151.748555 \mathrm{~nm}$. The lengths of slab 1 and 2 within a period are obtained from $a=\Lambda D$ and $b=\Lambda(1-D)$, respectively.

The DBR stack can be surrounded either by slab 1 and or by slab 2 . The total length of the grating is about $L_{\mathrm{DBR}} \approx 200 \mu \mathrm{m}$ and the number of periods is given by the integer quantum of $L_{\mathrm{DBR}} / \Lambda$. For $N=1$ and small duty cycles, the number of periods is 1313 . The $\kappa L$ value of the grating is about 5 for $N=1$ and $D=0.5$.

The aim of the modeling task is the computation of the reflectivity $R$ at $z=0$ of the fundamental guided transverse-electric (TE) mode and the corresponding value for the loss

$$
L=1-R-T
$$

with $T$ being the transmittivity at $z=L_{\mathrm{DBR}}$ versus the duty cycle $D$ for Bragg orders $N=1, \ldots, 3$.

\section{METHODS}

\section{A. Coupled-Mode Theory}

The coupled-mode equations for the amplitudes of the oppositely going waves can be written as [8]

$$
\pm \frac{\partial \psi^{ \pm}}{\partial z}=-i \Delta \beta \psi^{ \pm}-i \kappa \psi^{\mp}
$$

for a symmetric grating. The complex valued total coupling coefficient $\kappa$ is composed of terms due to the direct and indirect interactions of the two oppositely going waves with the grating [2], [5]. The indirect interaction gives also rise to a modification of both the real and imaginary parts of the relative propagation coefficient $\Delta \beta$ via the self-radiation coupling coefficient $\kappa_{\mathrm{s}}^{\mathrm{r}}$ given in the Appendix

$$
\Delta \beta=\frac{2 \pi n_{\mathrm{eff}}}{\lambda}-\frac{N \pi}{\Lambda}+i \kappa_{\mathrm{s}}^{\mathrm{r}} .
$$

Due to the fact that there is no gain and loss in the waveguide sections, the corresponding modal gain-loss function is omitted in (5).

It should be noted, that the CMT used here is different from the CMT applied to the modeling of optical waveguides, e.g., compare [9]. In the latter case, co- or contra-directional coupling of guided and radiation waveguide modes is considered and the CMT is here a rigorous method as far as a complete set of eigenmodes is taken as its basis.

In the case of Bragg waveguide gratings, typically only a single guided waveguide mode is considered which field distribution $E(x, z)$ is expressed as an infinite summation of partial waves dictated by the Bloch-Floquet theorem. Similarly, the dielectric function is expanded into a Fourier series. Due to the fact that only phase-matched terms are taken into account and the other approximations involved in the derivation [5], there are some restrictions in the applicability of (4), namely the conditions $|\kappa| \Lambda \ll 1$ and $|\Delta \beta| \Lambda \ll 1$ must hold [10]. These conditions mean that the perturbation of the field due to the grating has to be not too strong and that the wavelength of the field must be close to the Bragg condition. Note, that the CMT leads to multiple sets of equations, one for every Bragg order, but the direct solution of Maxwell's equations without recourse to the Bloch-Floquet theorem yields in principle a simultaneous description of all Bragg orders.

The reflection coefficient defined as $R=\left|\psi^{-}(0)\right|^{2} /\left|\psi^{+}(0)\right|^{2}$ is given by

$$
R=\left|\frac{-i \kappa \sin \left(\gamma L_{\mathrm{DBR}}\right)}{\gamma \cos \left(\gamma L_{\mathrm{DBR}}\right)+i \Delta \beta \sin \left(\gamma L_{\mathrm{DBR}}\right)}\right|^{2}
$$

and the transmission coefficient $T=\left|\psi^{+}\left(L_{\mathrm{DBR}}\right)\right|^{2} /\left|\psi^{+}(0)\right|^{2}$ by

$$
T=\left|\frac{\gamma}{\gamma \cos \left(\gamma L_{\mathrm{DBR}}\right)+i \Delta \beta \sin \left(\gamma L_{\mathrm{DBR}}\right)}\right|^{2}
$$

where

$$
\gamma=\sqrt{(\Delta \beta)^{2}-\kappa^{2}} .
$$

The inhomogeneous differential equations for the partial waves can be solved by different methods. In the original paper [2], the variation of parameters method was used. This approach was extended from four to multilayer waveguides in [11]. In [5], the equations were solved by means of the Green's function method using the exact one-dimensional Green's function of a multilayer waveguide and in addition to index gratings, gain and loss gratings were considered. Whereas in [5] only radiation fields were taken into account, in [2] and [11] 
evanescent fields which should not have a big impact on the results as revealed by this study were also included.

In [7], the Green's function method was also employed. However, the Green's function of a homogeneous unbounded medium was used. Furthermore, only second order gratings $(N=2)$ were considered and some unnecessary simplifications were made. In the Appendix, this approach is extended to higher Bragg orders and explicit expressions for the coupling coefficients and radiation losses are given.

\section{B. Bidirectional Mode Expansion}

In bidirectional mode expansion methods such as used in the tool CAMFR [12], the field in each slab is expanded onto eigenmodes in that slab. At the interfaces between the slabs, the transitions conditions give rise to reflection and transmission matrices. The propagation from one interface of the slab to the other interface results in diagonal propagation matrices.

For the determination of $R$ and $T$ of the entire structure, different algorithms can be used. A derivation and discussion of the so-called T-, S-, and R-matrix algorithms and variants can be found, e.g., in [13] and [14]. The T-matrix algorithm (also known as transfer matrix method) is the mathematically simplest algorithm. But it is numerically unstable due to the combination of falling and growing exponentials [13]. The derivation of the S-matrix algorithm which relates the incoming fields and the outgoing fields by means of scattering matrices is mathematically more involved. However, this algorithm can be implemented unconditionally stable. Similar holds for the R-matrix algorithm, but its implementation requires special treatment [14].

The advantage of mode expansion methods over FDTD and $\mathrm{MoL}$ consists in the fact, that no spatial discretization is required, which gives rise to only a small number of unknowns and therefore a fast model. One problem common to all methods is the proper treatment of the boundaries of the computational domain. Open boundaries are difficult to handle, but closed boundaries realized by electric or magnetic walls, e.g., lead to parasitic reflections. These reflections can be largely reduced by so-called perfectly matched layers (PMLs) placed in front of the walls. There exists different realizations of the PML boundary condition based on splitted fields [15], anisotropic media [16], or complex coordinate stretching [17].

In CAMFR, the $\mathrm{S}$-matrix algorithm is used for the calculation of $R$ and $T$. The structure is terminated by an electric wall on top and bottom. The PML boundary condition is implemented as proposed in [17]. For the simulation of the benchmark example, $100 \mathrm{TE}$ eigenmodes were used and the thicknesses of the PMLs have been chosen to $2 \mu \mathrm{m}$ with an imaginary part equals $-0.5 \mu \mathrm{m}$. These values establish a good compromise between a sufficient damping of residual reflections from the PMLs without influencing the fundamental guided mode too much and ensure energy conservation, nearly.

\section{RESULTS}

In this section, the reflectivity and the loss of the fundamental guided TE mode obtained by the tool CAMFR [12] and by CMT using the exact Green's function of a multilayer waveguide [5]

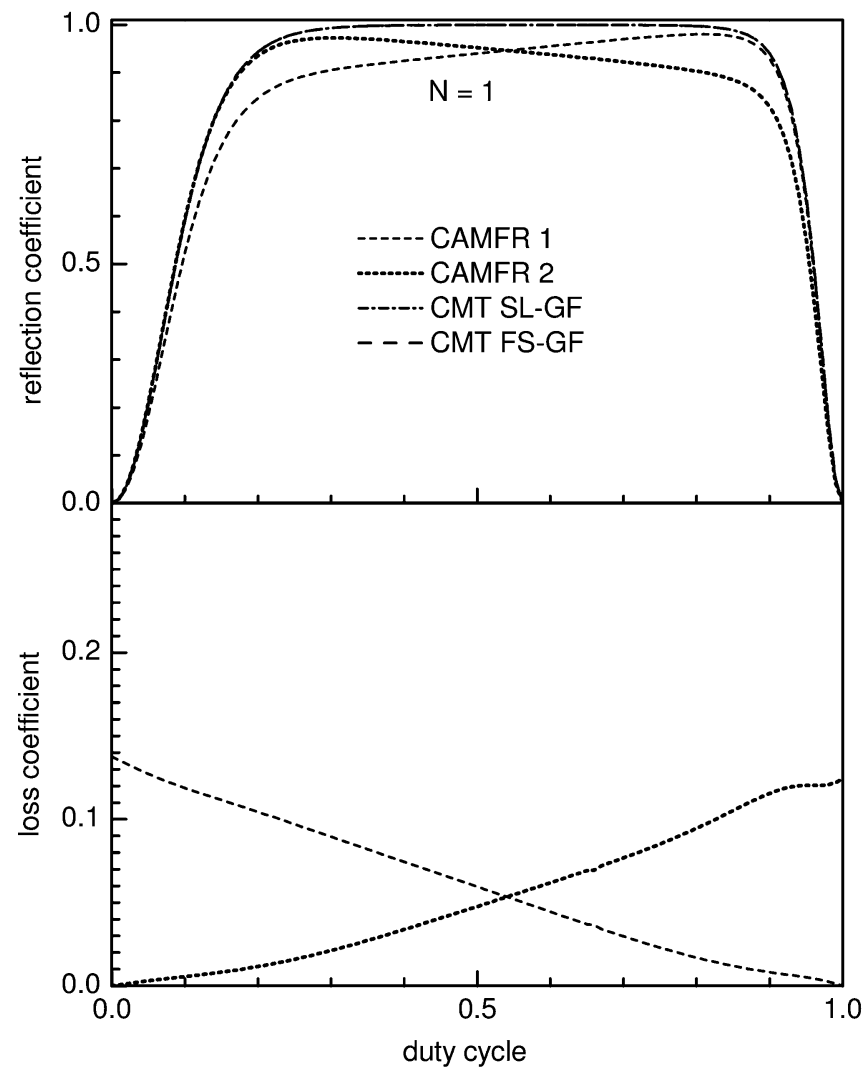

Fig. 2. Modal reflectivity (top) and corresponding modal loss (bottom) at the reference wavelength versus duty cycle for first-order grating. Short dashed (CAMFR 1): Mode expansion method with slab 1 enclosing the Bragg waveguide grating. Dotted (CAMFR 2): Mode expansion method with slab 2 enclosing the Bragg waveguide grating. Dash-dotted (CMT SL-GF): Coupled-mode theory using slab Green's function. Long dashed (CMT FS-GF): Coupled-mode theory using free-space Green's function.

as well as using the approximate free-space Green's function (see Appendix) are compared.

Fig. 2 depicts the results for the reflection coefficient and the corresponding values for the loss coefficient versus duty cycle of a first-order grating $(N=1)$ computed at the reference wavelength $\lambda=\lambda_{0} \equiv 980 \mathrm{~nm}$. The CMT yields vanishing loss for first-order gratings because there are no radiating fields. However, there is an insertion loss which is maximum for duty cycle $D=0$ if the Bragg grating is surrounded by slab 1 and for duty cycle $D=1$ if the Bragg grating is surrounded by slab 2 . The maximum insertion loss is almost twice the insertion loss of a single interface between the slabs $(L=0.07)$. The small differences in the losses for $D=0$ and $D=1$ are caused by residual reflections from the PMLs. The reflectivities obtained with CAMFR and CMT differ from those cases where the insertion loss is large. There is a good mutual agreement for small duty cycles and surrounding slab 2 as well as large duty cycles and surrounding slab 1.

In order to obtain the radiation loss with CAMFR, in the following figures, the insertion loss depicted in Fig. 2 is subtracted from the total loss obtained with CAMFR for the higher order gratings. Fig. 3 depicts the modal reflectivity and loss for a second order grating and Fig. 4 the results for a third order grating. The dependence of the reflection coefficient versus duty cycle, e.g., the position and values of maxima and minima is 


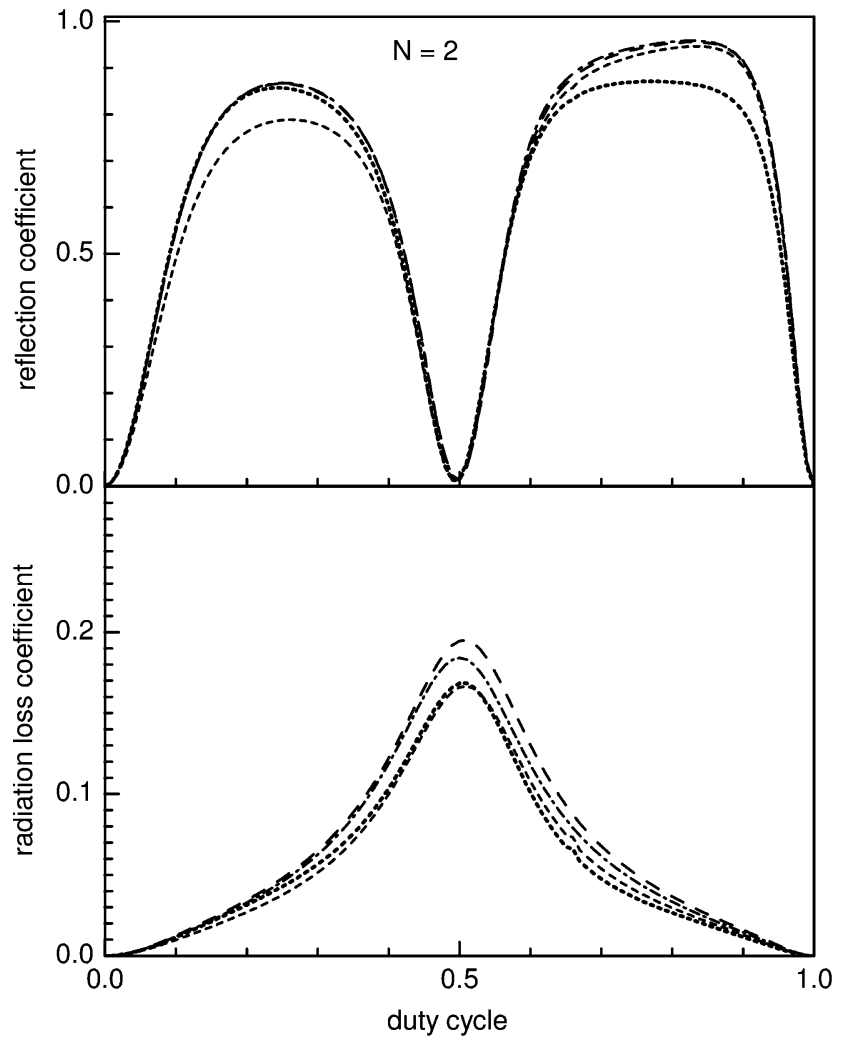

Fig. 3. (top) Modal reflectivity and (bottom) corresponding modal loss at the reference wavelength versus duty cycle for second order grating. The insertion loss from Fig. 2 was subtracted. Same lines as in Fig. 2.

well reproduced by all models. The increase of the reflection coefficient with duty cycle is also predicted by all models. There are larger deviations between CAMFR and CMT occurring for $D<0.5$ and surrounding slab 1 as well as for $D>0.5$ and surrounding slab 2 which are partially caused by the insertion losses.

There is also a surprisingly good correspondence in the radiation losses between CAMFR and CMT as well as between the two CMT models. The position of the maxima and minima of the loss coefficient is again well reproduced by all models. The small deviations between CAMFR and the CMT models are possibly caused partially by residual reflections, partially by the approximations inherent to the CMT models. The approximation of the Green's function by that of a homogeneous unbounded medium leads to a slight exaggeration of the radiation loss.

Figs. 5 and 6 depict exemplary spectra of the modal reflectivity and loss for a second order grating $(N=2)$ and duty cycles $D=0.25$ and $D=0.50$, respectively. The reflectivity spectra obtained with the different models agree very well for both duty cycles. Note, that the maximum of the reflectivity is not reached at the reference wavelength $\lambda_{0}=980 \mathrm{~nm}$, but at a different wavelength due to the influence of the radiation fields, i.e., due to the occurrence of $\kappa_{\mathrm{s}}^{\mathrm{r}}$ in (5). However, the deviation is very small $(|\Delta \lambda|<0.1 \mathrm{~nm})$.

For $D=0.25$, there is also a good agreement between the loss spectra in the vicinity of the maximum of the reflectivity and at longer wavelengths if the Bragg grating is surrounded by slab 2 because of the small insertion loss. However, there

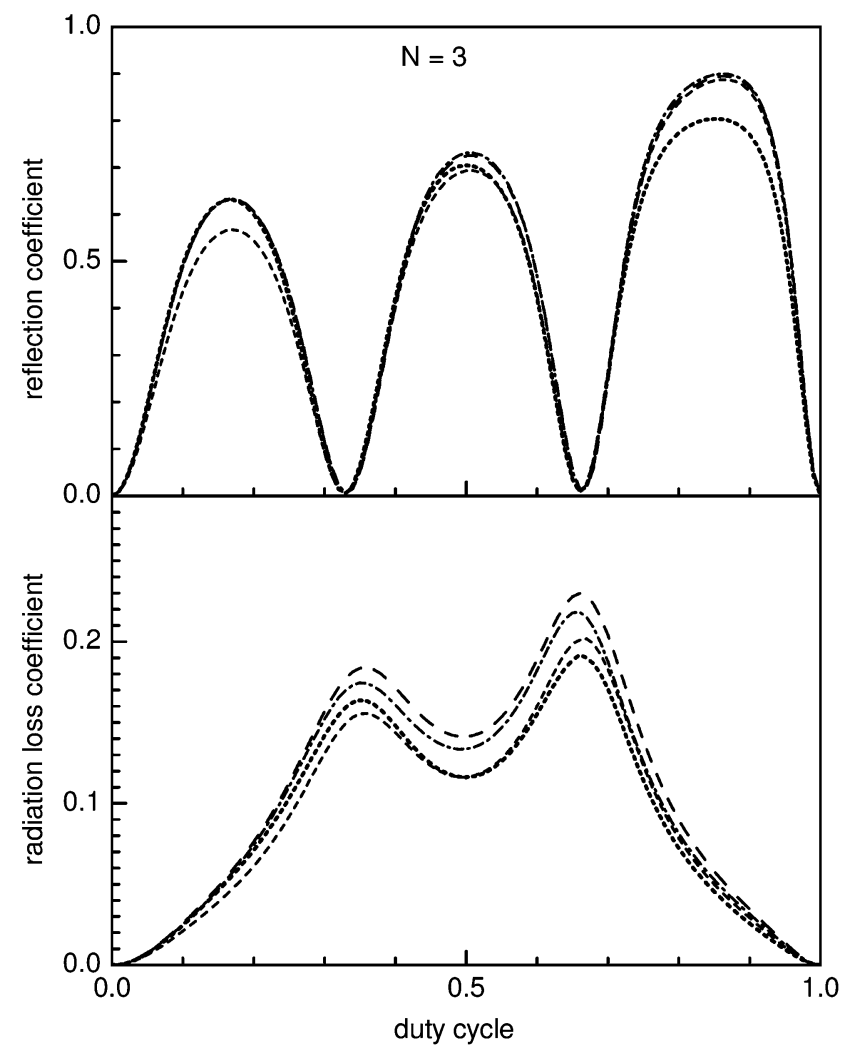

Fig. 4. (top) Modal reflectivity and (bottom) corresponding modal loss at the reference wavelength versus duty cycle for third order grating. The insertion loss from Fig. 2 was subtracted. Same lines as in Fig. 2.

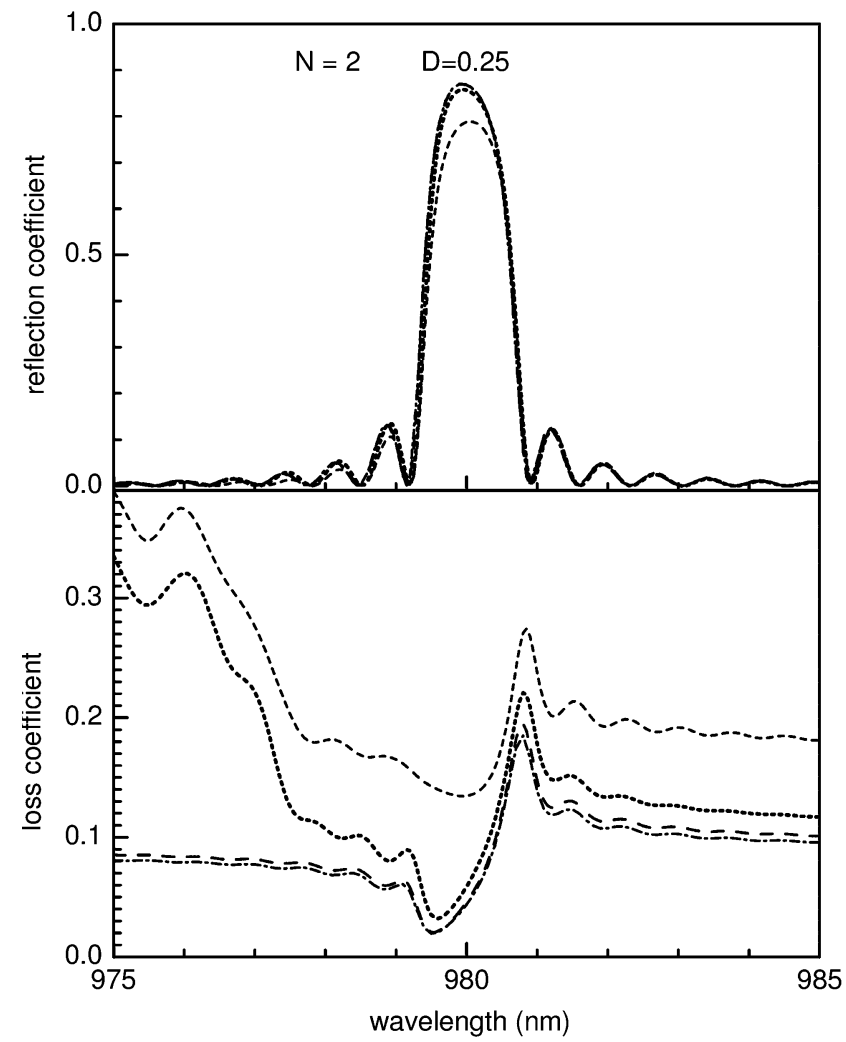

Fig. 5. (top) Modal reflectivity and (bottom) modal loss versus wavelength for second order grating and duty cycle 0.25 . Same lines as in Fig. 2.

is a large deviation between CAMFR and the CMT models at shorter wavelengths which is possibly caused by the appearance 


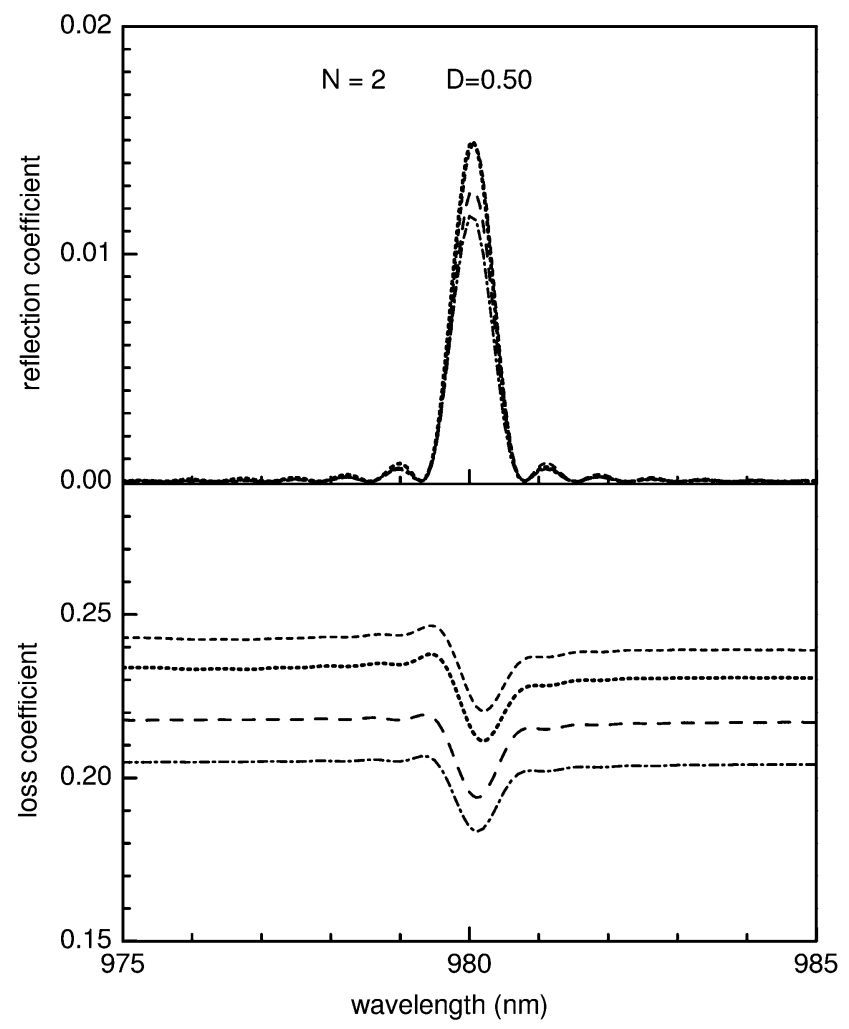

Fig. 6. (top) Modal reflectivity and (bottom) modal loss versus wavelength for second order grating and duty cycle 0.50 . Same lines as in Fig. 2.

of leaky Bloch-Floquet modes similarly as discussed in [3]. The different loss spectra for $D=0.50$ behave very similar, although the loss obtained with the CMT models is again slightly underestimated due to the insertion loss, at least partially (please note the scale of the ordinate).

\section{CONCLUSION}

The modal reflectivity and loss of lamellar diffraction gratings typically used in DFB and DBR lasers were computed using two CMT models and the tool CAMFR based on bidirectional mode expansion modeling. The good mutual agreement indicates the validity of the CMT as well as the capability of CAMFR to treat these Bragg waveguide gratings. The loss obtained with CAMFR includes additional insertion loss due to the mismatch between the field distributions in the grating and in the enclosing waveguides. This loss reduces also the reflectivity in certain cases.

The PML boundary condition as implemented in CAMFR models correctly the radiation fields. In one of the two CMT models the exact slab Green's function needed to compute the contribution due to the excited radiation fields for gratings with $N>1$ is approximated by that of a homogeneous unbounded medium. It turned out, that this approximation works very well.

\section{APPENDIX}

\section{FREE-SPACE GREEN's FUNCTION APPROXIMATION}

In this Appendix, explicit expressions for the coupling coefficients are given using the free space Green's function. We use partially the terminology of [5] and consider only TE modes. Hence, the main component of the electric field strength is $E_{y}$. The total coupling coefficient can be written as the sum of two terms

$$
\kappa=\kappa_{N}+i \kappa_{\mathrm{m}}^{\mathrm{r}}
$$

The first term $\kappa_{N}$ is due to the direct $N$ th order diffraction and is given by

$$
\kappa_{N}=\frac{k_{0}}{2 n_{\mathrm{eff}}} \int d x \xi_{N}(x) F^{2}(x)
$$

where $k_{0}=2 \pi / \lambda_{0}$ is the reference wave number of free space and $F(x)$ is the normalized profile of the considered guided mode of the reference waveguide. Here and in the following the integration extends from $-\infty$ to $+\infty$ if not otherwise indicated. The mutual-radiation coupling coefficient

$$
\kappa_{\mathrm{m}}^{\mathrm{r}}=\sum_{n=1}^{N-1} \kappa_{(n, N-n)}^{\mathrm{r}}
$$

arises from the indirect interaction via diffraction orders less than $N$. This interaction gives also rise to radiation loss

$$
\alpha^{\mathrm{r}}=-2 \operatorname{Re}\left(\kappa_{\mathrm{s}}^{\mathrm{r}}\right)
$$

where

$$
\kappa_{\mathrm{s}}^{\mathrm{r}}=\sum_{n=1}^{N-1} \kappa_{(n,-n)}^{\mathrm{r}}
$$

is the self-radiation coupling coefficient included in $\Delta \beta$ of (4). In (A3) and (A5)

$\kappa_{\left(n, n^{\prime}\right)}^{\mathrm{r}}=\frac{i k_{0}^{3}}{2 n_{\mathrm{eff}}} \int d x \int d x^{\prime} \xi_{n}(x) \xi_{n^{\prime}}\left(x^{\prime}\right) F(x) F\left(x^{\prime}\right) G_{\left|n^{\prime}\right|}\left(x, x^{\prime}\right)$.

In (A2) and (A6)

$$
\xi_{n}(x)=\Delta \epsilon(x) \frac{\sin \left(n \frac{\pi}{\Lambda} a(x)\right)}{n \pi}
$$

is the product of the perturbation $\Delta \epsilon$ of the dielectric function due to the grating and the $n$th Fourier coefficient of the grating. Note, that for a rectangular grating as considered in this paper $a(x)=$ constant and for the benchmark example furthermore $\Delta \epsilon(x)=$ constant inside and $\Delta \epsilon(x)=0$ outside the grating layer 6 hold.

The Green's function $G$ is a solution of the equation [5]

$\frac{\partial^{2} G_{n}\left(x, x^{\prime}\right)}{\partial x^{2}}+\left[\varepsilon(x) k_{0}^{2}-\frac{(N-2 n)^{2} \pi^{2}}{\Lambda^{2}}\right] G_{n}\left(x, x^{\prime}\right)=\delta\left(x-x^{\prime}\right)$.

In [5], (A8) was exactly solved by means of a transfer matrix method. Alternatively, the profile of the real valued dielectric function $\varepsilon(x)$ of the reference waveguide can be replaced by some average value $\bar{\varepsilon}$, for example by the effective index squared $n_{\text {eff }}^{2}$ of the reference waveguide. This approximation 
was firstly presented in [7] for second order gratings $(N=2)$ and later used for higher order gratings with any Bragg angle [18]. Here, the solution of (A8) is given by the outgoing Green's function of a homogeneous unbounded medium

$$
G_{n}\left(x, x^{\prime}\right)=-\frac{1}{2 i \tau_{n}} \begin{cases}e^{-i \tau_{n}\left(x-x^{\prime}\right)} & x>x^{\prime} \\ e^{-i \tau_{n}\left(x^{\prime}-x\right)} & x<x^{\prime}\end{cases}
$$

where

$$
\tau_{n}=k_{0} \sqrt{\bar{\varepsilon}-\frac{(N-2 n)^{2} \pi^{2}}{k_{0}^{2} \Lambda^{2}}} .
$$

Note, that the temporal variation of the field is given by $\exp \left(+i k_{0} c t\right)$. The Bragg period in (A10) can be expressed in terms of $n_{\text {eff }}$ and $N$ according to (1). If we insert (A9) into (A6) and split the exponential function into sinus and cosinus terms, we obtain

$$
\begin{aligned}
\kappa_{\left(n, n^{\prime}\right)}^{\mathrm{r}}= & -\frac{k_{0}^{3}}{4 n_{\mathrm{eff}} \tau_{\left|n^{\prime}\right|}} \int d x \xi_{n}(x) F(x) \\
& \times\left\{\int d x^{\prime} \xi_{n^{\prime}}\left(x^{\prime}\right) F\left(x^{\prime}\right) \cos \left(\tau_{\left|n^{\prime}\right|}\left(x^{\prime}-x\right)\right)\right. \\
& +i \int_{-\infty}^{x} d x^{\prime} \xi_{n^{\prime}}\left(x^{\prime}\right) F\left(x^{\prime}\right) \sin \left(\tau_{\left|n^{\prime}\right|}\left(x^{\prime}-x\right)\right) \\
& \left.-i \int_{x}^{+\infty} d x^{\prime} \xi_{n^{\prime}}\left(x^{\prime}\right) F\left(x^{\prime}\right) \sin \left(\tau_{\left|n^{\prime}\right|}\left(x^{\prime}-x\right)\right)\right\}
\end{aligned}
$$

Note, that in contrast what was stated in [7], the sinus terms do not cancel, which was already recognized in [19]. Actually, only by keeping these terms $\kappa_{\left(n, n^{\prime}\right)}^{\mathrm{r}}$ possesses an imaginary part. Equation (A11) can be transformed to

$$
\begin{aligned}
\kappa_{\left(n, n^{\prime}\right)}^{\mathrm{r}}= & -\frac{k_{0}^{3}}{4 n_{\mathrm{eff}} \tau_{\left|n^{\prime}\right|}}\left\{\int d x \xi_{n}(x) F(x) \cos \left(\tau_{\left|n^{\prime}\right|} x\right)\right) \\
& \times \int d x \xi_{n^{\prime}}(x) F(x) \cos \left(\tau_{\left|n^{\prime}\right|} x\right) \\
& +\int d x \xi_{n}(x) F(x) \sin \left(\tau_{\left|n^{\prime}\right|} x\right) \\
& \left.\times \int d x \xi_{n^{\prime}}(x) F(x) \sin \left(\tau_{\left|n^{\prime}\right|} x\right)\right\} \\
& +\frac{i k_{0}^{3}}{4 n_{\mathrm{eff}} \tau_{\left|n^{\prime}\right|}}\left\{\int d x \xi_{n}(x) F(x) \cos \left(\tau_{\left|n^{\prime}\right|} x\right)\right. \\
& \times \int d x \xi_{n^{\prime}}(x) F(x) \sin \left(\tau_{\left|n^{\prime}\right|} x\right) \\
& \left.-\int d x \xi_{n}(x) F(x) \sin \left(\tau_{\left|n^{\prime}\right|} x\right)\right) \\
& \times \int d x \xi_{n^{\prime}}(x) F(x) \cos \left(\tau_{\left|n^{\prime}\right|} x\right) \\
& -2 \int d x \xi_{n}(x) F(x) \cos \left(\tau_{\left|n^{\prime}\right|} x\right) \\
& \times \int_{-\infty}^{x} d x^{\prime} \xi_{n^{\prime}}\left(x^{\prime}\right) F\left(x^{\prime}\right) \sin \left(\tau_{\left|n^{\prime}\right|} x^{\prime}\right)
\end{aligned}
$$

$$
\begin{aligned}
& \left.+2 \int d x \xi_{n}(x) F(x) \sin \left(\tau_{\left|n^{\prime}\right|} x\right)\right) \\
& \left.\times \int_{-\infty}^{x} d x^{\prime} \xi_{n^{\prime}}\left(x^{\prime}\right) F\left(x^{\prime}\right) \cos \left(\tau_{\left|n^{\prime}\right|} x^{\prime}\right)\right\}
\end{aligned}
$$

more suitable for a numerical evaluation. Note, that if $\xi_{n}(x)$ is constant it can be drawn out of the integrals and $\kappa_{\left(n, n^{\prime}\right)}^{\mathrm{r}}$ can be evaluated analytically.

\section{ACKNOWLEDGMENT}

One of the authors, H. Wenzel, would like to thank M. Wienold for supporting the computations.

\section{REFERENCES}

[1] H. Kogelnik and C. V. Shank, "Coupled mode theory of distributed feedback lasers," J. Appl. Phys., pp. 2327-2335, 1972.

[2] W. Streifer, D. Scifres, and R. D. Burnham, "Coupled wave analysis of DFB and DBR lasers," IEEE J. Quantum Electron., vol. 13, no. 4, pp. 134-141, Apr. 1977.

[3] J. Ctyroky, S. Helfert, R. Pregla, P. Bienstmann, R. Baets, R. D. Ridder, R. Stoffer, G. Klaasse, J. Petracek, P. Lalanne, J.-P. Hugonin, and R. M. D. L. Rue, "Bragg waveguide grating as a 1-D photonic bandgap structure: COST 268 modeling task," Opt. Quantum Electron., vol. 34, pp. $455-470,2002$.

[4] G. R. Hadley, "Low-truncation-error fnite difference representations of the 2-d helmholtz equation," Int. J. Electron. Commun., vol. 52, pp. 310-316, May 1998.

[5] A. M. Shams-Zadeh-Amiri, J. Hong, X. Li, and W. P. Huang, "Second and higher resonat gratings with gain or loss-Part I: Green's function analysis," IEEE J. Quantum Electron., vol. 36, no. 12, pp. 1421-1430, Dec. 2000.

[6] P. Bienstman and R. Baets, "Optical modeling of photonic crystals and VCSELs using eigenmode expansion and perfectly matched layers," Opt. Quantum Electron., vol. 33, pp. 327-341, Apr. 2001.

[7] R. Kazarinov and C. H. Henry, "Second-order distributed feedback lasers with mode selection provided by first-order radiation losses," IEEE J. Quantum Electron., vol. 21, no. 2, pp. 144-150, Feb. 1985.

[8] H. Wenzel, "Green's function based simulation of the optical spectrum of multisection lasers," IEEE J. Quantum Electron., vol. 9, no. 3, pp. 865-871, Mar. 2003.

[9] D. Marcuse, "Coupled mode theory," in Theory of Dielectric Optical Waveguides. Norwell, MA: Academic Press, 1991, ch. 3.

[10] U. Bandelow, "Theorie longitudinaler effekte in $1.55 \mu \mathrm{m}$ mehrsektions-DFB-laserdioden," Ph.D. dissertation, Humboldt-Universität, Berlin, Germany, 1994.

[11] J. Kinoshita, "Analysis of radiation mode effects on oscillating properties of DFB lasers," IEEE J. Quantum Electron., vol. 35, no. 11, pp. 1569-1583, Nov. 1999.

[12] CAMFR - CAvity Modeling FRamework, P. Bienstman, L. Vanholme, M. Ibanescu, P. Dumon, and R. Baets. [Online]. Available: http://camfr.sourceforge.net/

[13] L. Li, "Multilayer modal method for diffraction gratings of arbitrary profile, depth, and permittivity," J. Opt. Soc. Amer. A, vol. 10, pp. 2581-2591, 1993

[14] — - "Formulation and comparison of two recursive matrix algorithms for modeling layered diffraction gratings," J. Opt. Soc. Amer. A, vol. 13, pp. 1014-1035, 1996.

[15] J.-P. Berenger, "A perfectly matched layer for the absorption of electromagnetic waves," J. Comput. Phys., vol. 114, pp. 185-200, Oct. 1994.

[16] Z. S. Sacks, D. K. Kingsland, and R. Lee, "A perfectly matched anisotropic absorber for use as an absorbing boundary condition," IEEE Trans. Antennas Propagat., vol. 43, no. 12, pp. 1460-1463, Dec. 1995.

[17] W. C. Chew, J. Jin, and E. Michielssen, "Complex coordinate stretching as a generalized absorbing boundary condition," Microw. Opt. Technol. Lett., vol. 15, pp. 363-369, Jun. 1997.

[18] R. Güther, "Abstrahlungsverluste bei bragg-gitter-beugung höherer ordnung in wellenleitern," in Proc. 105. DGaO Jahrestagung, 2004, http://www.dgao-proceedings.de, p. 29.

[19] LASTIP User's Manual, Crosslight Software Inc., 2003. 
Hans Wenzel received the Diploma and Doctoral degrees in physics from Humboldt-University, Berlin, Germany, in 1986 and 1991, respectively. His dissertation dealt with the electrooptical modeling of semiconductor lasers.

From 1991 to 1994, he was involved in a research project on the three-dimensional simulation of DFB lasers. In 1994, he joined the Ferdinand-Braun-Institut für Höchstfrequenztechnik, Berlin, Germany, where he is engaged in the development of high-power semiconductor lasers. His main research interests include the analysis, modeling, and simulation of optoelectronic devices.

Reiner Güther received the Diploma and the Dr.rer.nat. degree from University of Jena, Jena, Germany, in 1964 and 1967, respectively, the Dr.Sc. degree from Academy of Sciences of Berlin in 1976, the Facultas Docendi from the Technical University Ilmenau, Ilmenau, Germany, in 1982, and the Dr.habil. degree from University Leipzig, Leipzig, Germany, in 1993.

During 1968-1991, he worked in the Central Institute of Optics and Spectroscopy (ZOS), Berlin of the Academy of Sciences, Berlin, Germany, partially as Department Head of Applied Optics. Since 1992, he has been a Researcher in the Ferdinand-Braun-Institut für Höchstfrequenztechnik, Berlin. His research activities include quantum field theory, nonlinear optics, volume holography, electro-optics, electromagnetic phenomena and correction theory of holographic concave gratings, diffractive optics, and waveguide coupling, mostly by direct order of optics industry, like Carl Zeiss or Telekom. His current research activities are the design of semiconductor lasers with planar filtering structures, and laser optics, including micro-optics.

Dr. Güther is a member of $\mathrm{DGaO}$ and EOS.
Ali M. Shams-Zadeh-Amiri (M'00) received the B.S. and M.S. degrees in electrical engineering from the University of Tehran, Tehran, Iran, in 1987 and 1990, respectively, and the Ph.D. degree in electrical engineering from University of Waterloo, Waterloo, ON, Canada, in 1997. His doctoral research focused on surface-emitting distributed feedback (DFB) lasers with circular and rectangular gratings.

Until 1998, he was with Nortel Networks, Ottawa, ON, Canada, where he conducted research and development of multiple quantum well gain-coupled DFB lasers with second-order gratings for advanced optical communications systems. From 1999 to 2000, he was with the Department of Electrical and Computer Engineering, University of Waterloo as a Research Assistant Professor, conducting research on dielectric resonators and teaching courses on electromagnetic theory. In 2000, he joined PhotoNami Inc., Richmond Hill, Canada, as a Research Scientist. Currently, he is the leader of the laser team and conducting research and development of multiwavelength laser sources for optical switches in metropolitan area networks. He has four U.S. patents pending. His research interests include multiple quantum well DFB lasers in general and surface-emitting DFB lasers, in particular integrated optics, numerical simulation of optoelectronic devices, fiber gratings, microwave planar structures, dielectric resonators, and electromagnetic theory.

Peter Bienstman was born in Ghent, Belgium, in 1974. He received the M.S. degree in electrical engineering in 1997 and the Ph.D. degree in 2001 from the Department of Information Technology (INTEC), Ghent University, Belgium.

$\mathrm{He}$ is currently an Assistant Professor at Ghent University. During 2001-2002, he spent a year in the Joannopoulos research Group at the Massachusetts Institute of Technology (MIT). His research interests include the modeling of optical structures, notably photonic crystal structures, vertical-cavity surface-emitting lasers, and resonant-cavity light-emitting diodes. $\mathrm{He}$ has published several papers and has one patent.

Dr. Bienstman a member of IEEE LEOS. 